\title{
Attenuation of Low Ambient Temperature- Induced Myocardial Hypertrophy by Atorvastatin via Promoting Bcl-2 Expression
}

\author{
Jing Liang ${ }^{\mathrm{a}, \mathrm{d}}$ Kun Yinc Xuefeng Cao ${ }^{\mathrm{e}}$ Zhenbo Han $^{\mathrm{a}}$ Qi Huang ${ }^{\mathrm{a}}$ Lai Zhang $^{\mathrm{a}}$ \\ Wenya Ma ${ }^{a}$ Fengzhi Ding ${ }^{a}$ Chongwei Bia Dan Feng ${ }^{a}$ Yu Liu ${ }^{b}$ Zhenwei Pan ${ }^{a}$ \\ aDepartment of Pharmacology, College of Pharmacy, Harbin Medical University, Harbin, Heilongjiang \\ Province, ${ }^{b}$ Clinical Laboratory Department of the Fourth Affiliated Hospital of Harbin Medical \\ University, Harbin, Heilongjiang Province; 'Affiliated Second Hospital of Harbin Medical University, \\ Harbin, Heilongjiang Province, 'Tianjin Medical University, Tianjin, eDepartment of Anesthesiology, \\ Affiliated Hospital of Chengde Medical University, Chengde, Hebei Province, China
}

\section{Key Words}

Atorvastatin $\cdot$ Heart $\cdot$ Cold $\cdot \mathrm{p} 38 \cdot \mathrm{Bcl}-2 \cdot$ Cardiac hypertrophy

\begin{abstract}
Background/Aims: It is well documented that myocardial hypertrophy is associated with low ambient temperature. Atorvastatin (Atv) has been shown to protect against atherosclerosis, cardiac fibrosis, ischemia/reperfusion injury, etc. In this study, we aim to determine whether atorvastatin is effective in the treatment of myocardial hypertrophy induced by cold exposure and to shed light on underlying mechanism. Methods: The mice aged 4-week were randomized to Control (Ctl) group (raised at room temperature), Cold group (raised at $3-5^{\circ} \mathrm{C}$ ) and Atv treatment group (raised at $3-5^{\circ} \mathrm{C}$ followed by $10 \mathrm{mg} / \mathrm{kg} /$ day Atv infusion). Echocardiography (ECG), HE, TUNEL and Masson's trichrome staining, and Transmission electronic microscopy were performed to analyze cardiac function, myocardial hypertrophy, cardiac fibrosis, apoptosis and cardiomyocyte ultrastructure, respectively. Western blot was carried out to determine the involvement of MAPK and apoptosis pathways. Results: Exposure of mice to low temperature induced myocardial hypertrophic growth characterized by the elevation of heart/body weight index and heart weight /tibia length index, compared with control mice. Atv treatment attenuated cardiac hypertrophy induced by cold exposure; Atv also attenuated the increase of cross-sectional area of cardiomyocytes and cardiac collagen content fraction in mice exposed to cold. ECG showed that the decline of cardiac functions including the elevated left ventricular systolic/diastolic internal dimension (LVIDs/d) and fractional shortening (FS) in mice with cold exposure was also inhibited by Atv treatment. Transmission electronic microscopy uncovered that Atv attenuated mitochondrial injury induced by cold exposure in mice. In addition, systolic blood pressure was gradually increased in mice exposed to cold
\end{abstract}

J. Liang, K. Yin and X. Cao made equal contribution to this work.

Prof. Zhenwei Pan and Yu Liu

KARGER
Department of Pharmacology, Harbin Medical University, Harbin 150081, Heilongjiang Province and Clinical Laboratory, the Fourth Affiliated Hospital of Harbin Medical University, Harbin 150001, Heilongjiang Province (China)

Tel. +86 451 86671354, E-Mail panzw@ems.hrbmu.edu.cn 
temperature, and Atv treatment significantly inhibited the elevation of blood pressure in cold-treated mice. Mechanistically, mitogen-activated protein kinase (MAPK) signal was not altered in mice exposed to cold, and Atv did not affect MAPK signal in cold-treated mice. But Atv mitigated the reduction of $\mathrm{Bcl}-2 / \mathrm{Bax}$ level in heart of cold-treated mice. Conclusion: Atv attenuated myocardial hypertrophy induced by cold exposure through inhibiting the downregulation of $\mathrm{Bcl}-2$ in heart. It may provide a novel strategy for low temperature-induced myocardial hypertrophy treatment.

\section{Introduction}

Cardiovascular disease (CVD) is one of the most common causes of death worldwide. The mortality and morbidity resulting from CVD is growing yearly. Cardiac hypertrophy is myocardial dilation in response to various stimuli [1]. Though physiological hypertrophy is beneficial to maintain cardiac functions, long-standing pathologic hypertrophy will result in dysregulation of myocardial contraction and heart failure eventually which largely contributes to growing mortality and morbidity of CVD [2]. A lot of stimuli are associated with cardiac hypertrophy and heart failure, such as long-term hypertension, heart infarction as well as valvular disease [3-6]. Low ambient temperature is capable of promoting constriction of blood vessels to maintain normal body temperature. Resultantly, peripheral vascular resistance is increased which results in elevation of blood pressure as well as myocardial oxygen consumption. To date, substantial studies have demonstrated that low ambient temperature is a risk factor of cardiac hypertrophy [7-9]. Hypertension, an important trigger of cardiac hypertrophy, frequently happened at low temperature [10-12]. Besides, low temperature induces CVD through interrupting many molecules and pathways implicating in CVD, such as endothelin-1 (ET-1), nitric oxide (NO), sympathetic nervous system and renin-angiotensin system (RAS). Interestingly, they are also involved in cardiac hypertrophy [13-16]. Therefore, low temperature can induce cardiac hypertrophy via various mechanisms.

Atv, a member of stains lowering cholesterol in blood by inhibiting HMG-CoA reductase, alleviates CVD and blood cholesterol dysregulation effectively. It markedly lowers blood cholesterol by regulating lipoprotein-cholesterol complex and liver function $[17,18]$. Atv has been applied to treat CVD, atherosclerosis and other pathophysiological processes through modulating endothelial cell function as well as other pathways $[19,20]$. Though it is considerably reported that Atv can treat CVD, its role in cold-induced cardiac hypertrophy as well as the underlying mechanism remains largely unknown. Thus, our study aimed to unveil the effect of Atv on low temperature-induced cardiac hypertrophy.

\section{Material and Methods}

Animals

The Kunming adult male mice used in this study were purchased, raised and grouped as previous study [7]. 10mg/kg Atv was given to mice by gastric gavage in Atv group, and Ctl as well as Cold groups were treated with isovolumic PBS. The animal protocol was approved by the Institutional Animal Care and Use Committee of Harbin Medical University. All the procedures were in agreement with the Directive 2010/63/ EU of the European Parliament.

\section{Echocardiography}

Mice were anesthetized with ketamine $(100 \mathrm{mg} / \mathrm{kg})$ and xylazine $(10 \mathrm{mg} / \mathrm{kg})$ followed by echocardiography (ECG) according to previous study [21]. HP Sonos 2500 (Hewlett-Packard, New Orleans, LA, USA) equipped with a 30-MHz phased-array transducer was applied to detect cardiac function. Here, we determined LVPWd (diastolic left ventricular posterior wall thickness), LVPWs (systolic left ventricular posterior wall thickness), LVIDd (diastolic left ventricular internal dimensions ) and LVIDd (diastolic 


\section{Cellular Physiology Cell Physiol Biochem 2017;41:286-295

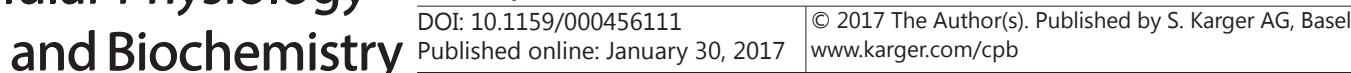 \\ Liang et al.: Atorvastatin Inhibited Cold-Induced Hypertrophy}

left ventricular internal dimensions) using M mode acquisition. EF (ejection fraction) and FS (fraction shortening ) were calculated as previous studies [22]. All parameters of cardiac function were obtained by calculating 3 successive cardiac cycles and analyzed by ECG expert.

\section{Blood pressure}

Tail-cuff was performed to determine blood pressure. BP-2000 Blood Pressure Analysis System (Visitech Systems, Cary, NC) was applied to record systolic blood pressure. Mice were secured in rodent restrictor followed by systolic blood pressure recording for 10 times successively. The data could be applied for further analysis as previous study [7].

\section{Stainings}

After 8-week, mice were sacrificed followed by formalin fixation and paraffin embedding. Next, left ventricle of heart was sectioned and stained with HE, Masson's trichrome and TUNEL staining according to our previous study [21]. Subsequently, sections were observed using fluorescence microscope. HE, Masson's trichrome and TUNEL staining were applied to determine myocardial structure, fibrosis and cell apoptosis respectively. All acquisitions were analyzed with Image-pro-plus 6.0 software.

Transmission electron microscopy

Transmission electron microscopy was performed to observe ultrastructural alterations of cardiomyocyte. Hitachi H-7650 transmission electron microscopy was applied for observation.

\section{Western blot}

Western blot was performed to determine protein level. Proteins were extracted from tissue obtained from left ventricle. Proteins associated with mitogen-activated protein kinase (MAPK) and apoptosis pathways were determined. In our case, samples were resolved using $10 \%$ and $12 \%$ polyacrylamide gels followed by protein samples transfer, primary and secondary antibody incubation. At last, membranes were analyzed with Odyssey Infrared Imaging System (LI-COR Bioscience, Lincoln, NE). The captures were analyzed with Image J software.

\section{Statistics}

All data were represented as mean \pm SEM. $P$ values were calculated with one-way ANOVA followed by Bonferroni by using Graphpad Prism software unless described otherwise. $P<0.05$ indicated statistical significance.

\section{Results}

Atv attenuated cardiac hypertrophy induced by cold exposure

After 8-week, mice were sacrificed followed by body weight, heart weight and tibial length measurement. In comparison with Ctl, the ratios of heart weight/body weight and

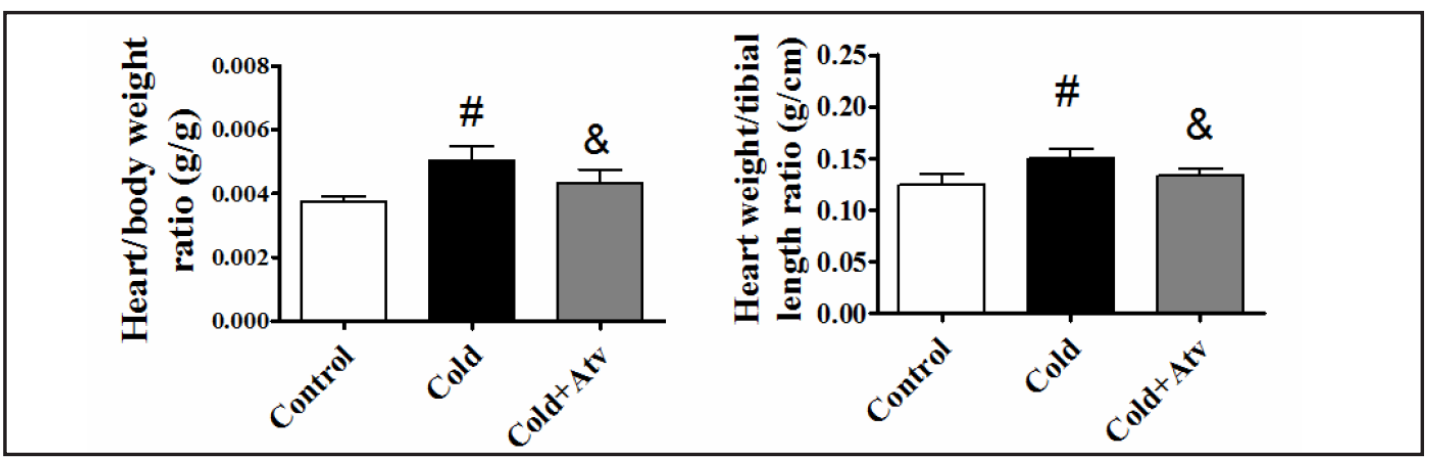

Fig. 1. Effect of Atv on hearts size of mice exposed to cold. (A), The ratio of HW/BW of Ctl, Cold and Atv groups showed Atv attenuated HW/BW increase caused by cold exposure significantly; (B), The ratio of HW/TL showed Atv attenuated HW/TL increase caused by cold exposure as well. Results are showed as mean \pm SEM. \# $\mathrm{p}<0.05$ vs Ctl, $\& \mathrm{p}<0.05$ vs Cold, $\mathrm{n}=6$. 


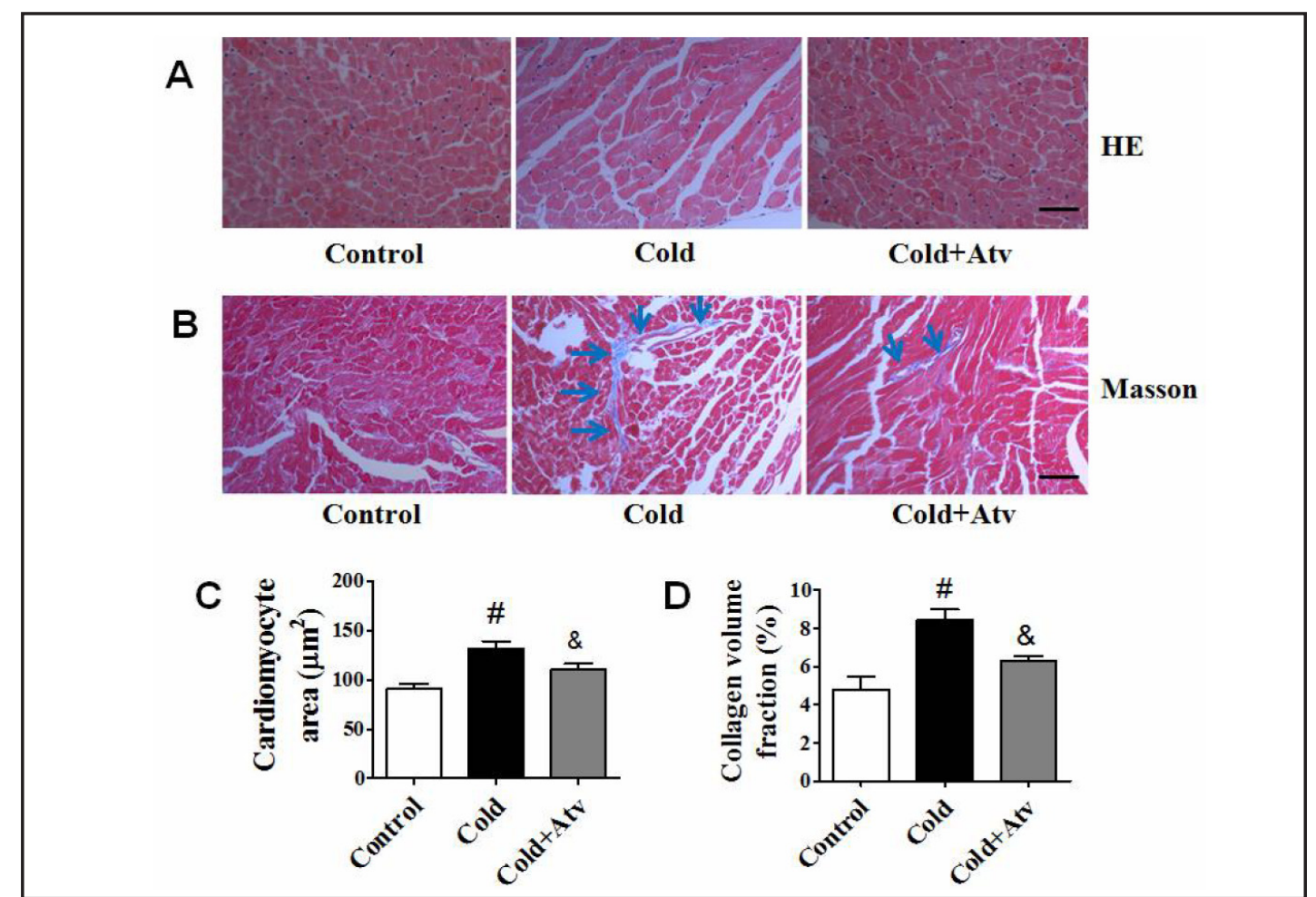

Fig. 2. Effect of Atv on the structure of hearts of mice exposed to cold. (A), HE staining of heart tissue to evidence changes of cross-sectional area. It indicated Atv was capable of attenuating cardiomyocytes hypertrophy induced cold exposure; (B), Masson's trichrome staining of heart tissue to show interstitial collagen content of each group. It indicated Atv was capable of attenuating interstitial collagen content increase induced by cold exposure; (C and D), Statistics of A and B analyzed by Image Pro. They showed similar and significant results with A and B. The scale bar: $50 \mu \mathrm{m}$. Results are showed as mean $\pm \mathrm{SEM}$. \# p<0.05 vs Ctl, $\& \mathrm{p}<0.05$ vs Cold.

heart weight/tibial length were significantly higher in cold-exposed group while Atv lowered both (Fig. 1). It indicated that Atv was capable of attenuating cardiac hypertrophy induced by cold exposure.

Atv attenuated cardiac fibrosis and cardiomyocytes hypertrophy induced by cold exposure Heart tissue was sectioned and stained with HE as well as Masson's trichrome staining to observe pathological changes of cardiac structure (Fig. 2). Figure 2A showed that the cross-sectional area of cardiomyocytes increased after cold exposure while Atv could reverse this. It indicated that Atv was capable of attenuating cardiomyocyte hypertrophy induced by cold exposure. Correspondingly, statistics of cross-sectional area in Figure 2C showed that Atv was capable of inhibiting cardiomyocyte hypertrophy significantly induced by cold exposure. Cardiac fibrosis was detected which was showed in Figure 2B and 2D. Figure 2B manifested that cardiac fibrosis increased significantly after cold exposure while Atv decreased it. Figure 2D showed statistics of interstitial fibrosis in heart. Atv treatment greatly suppressed the increase of cardiac fibrosis in cold-treated mice. It was consistent with Figure 2B.

Atv attenuated ultrastructural damage of cardiomyocytes induced by cold exposure

Cardiac hypertrophy was frequently accompanied with ultrastructural damage of cardiomyocytes. Transmission electron microscopy was employed to detect these changes. Figure 3 evidenced that myofilament broken, mitochondrial autophagy as well 


\section{Cellular Physiology and Biochemistry

Fig. 3. Effect of Atv on ultrastructure of cardiomyocytes exposed to cold. It manifested that mitochondrial autophagy and swelling accompanied with myofilament broken happened in cardiomyocytes of mice exposed to cold while Atv treatment was able to reverse them. The scale bar: $1 \mu \mathrm{m}$. $\boldsymbol{\Delta}$ indicated mitochondrial autophagy; $\bullet$ indicated mitochondrial swelling; $\star$ indicated myofilament broken.

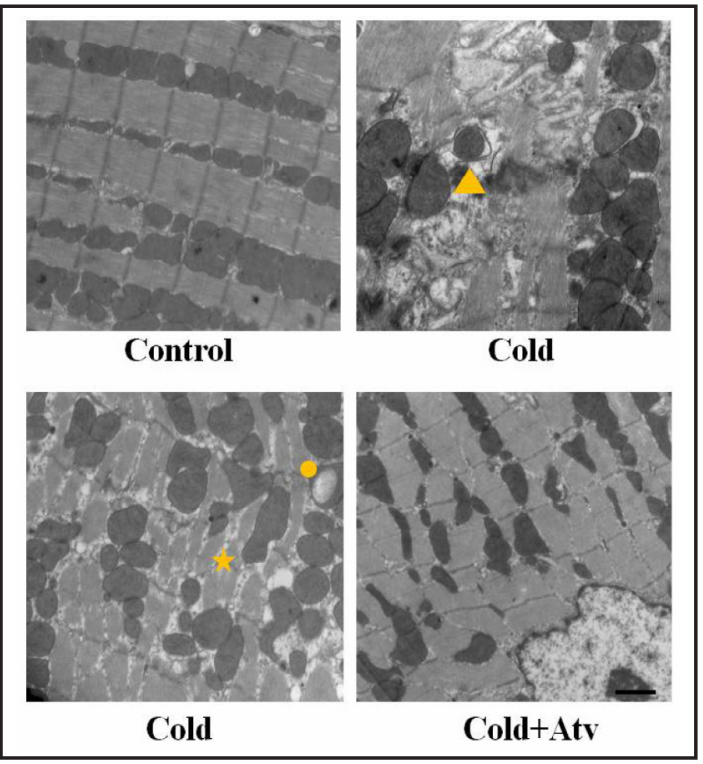

A

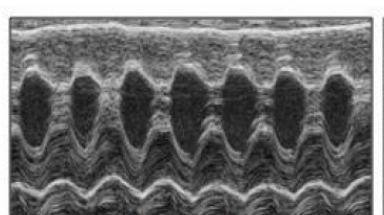

Control
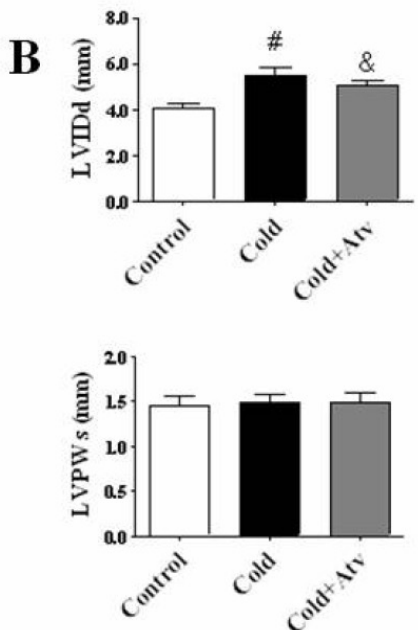

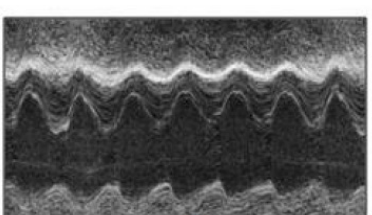

Cold
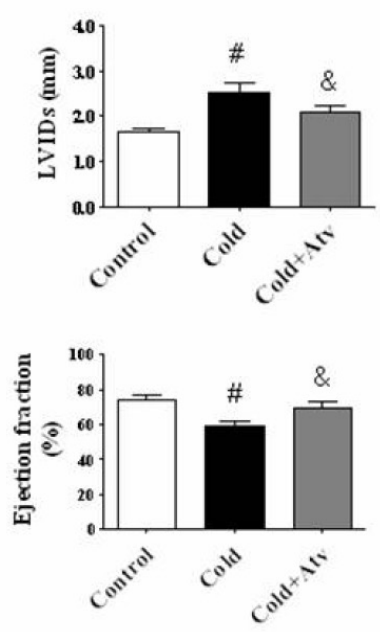

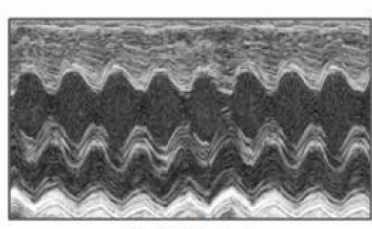

Cold+Atv
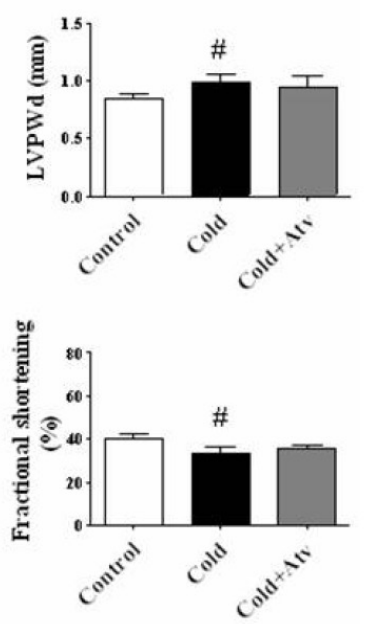

Fig. 4. Effect of Atv on cardiac function of mice exposed to cold. (A), Representative echocardiography of Ctl, Cold and Atv groups. It indicated that Atv was capable of decreasing volume of LV elevated by cold exposure; (B), Statistics of echocardiography. It indicated that cold exposure was capable of decreasing EF while increasing LVIDs/d. However, these conditions were mitigated by Atv, and thus protecting heart. Results are showed as mean \pm SEM. \# $\mathrm{p}<0.05$ vs Ctl, \& $\mathrm{p}<0.05$ vs Cold, $\mathrm{n}=6$.

as mitochondrial swelling occurred in cardiomyocytes of mice exposed to cold while these damage were greatly ameliorated after Atv administration. Therefore, Atv could protect cardiomyocytes from ultrastructural damage resulting from cold exposure.

\section{KARGER}




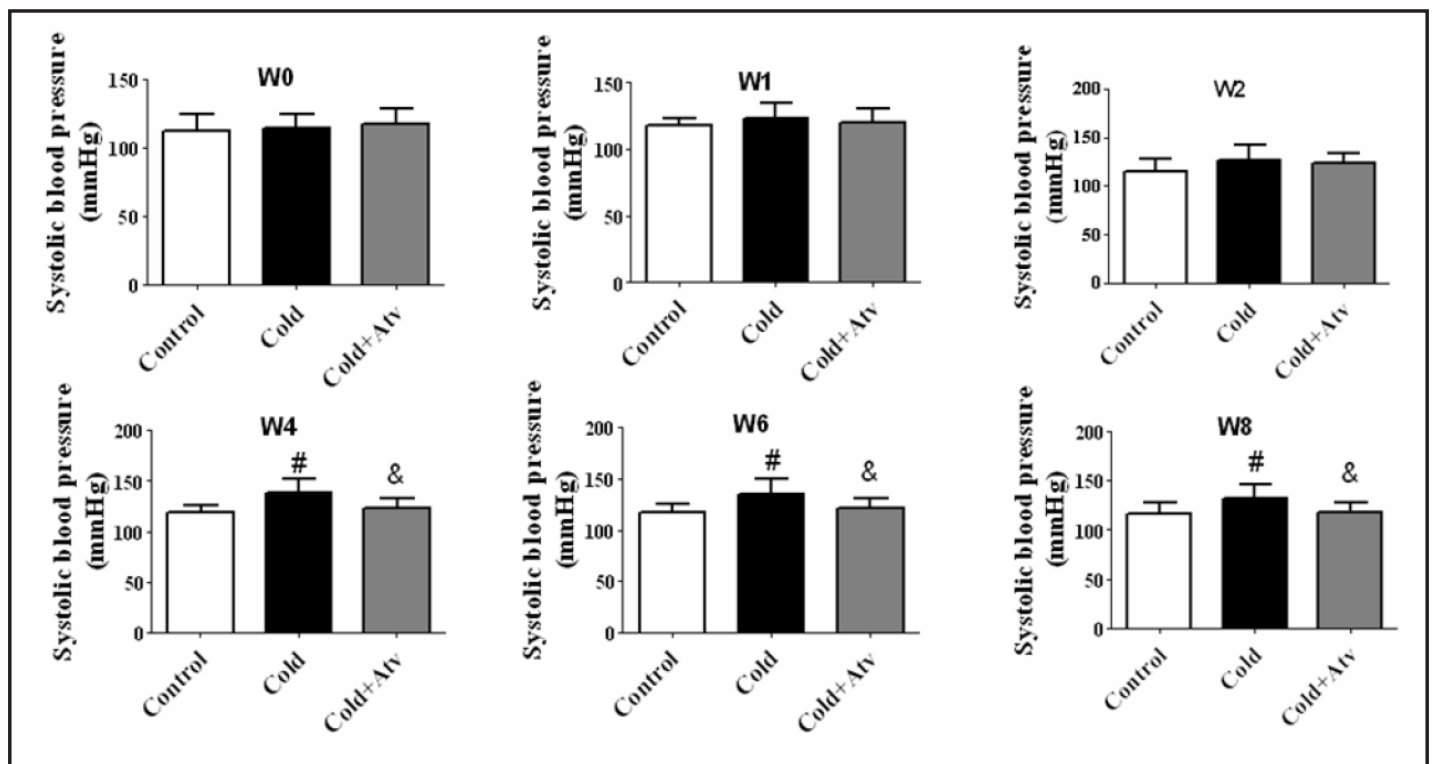

Fig. 5. Effect of Atv on blood pressure of mice exposed to cold. The figure showed the systolic blood pressure of each group. It indicated that Atv was capable of lowering blood pressure of mice exposed to cold. Results are showed as mean \pm SEM. \# $\mathrm{p}<0.05$ vs Ctl, $\& \mathrm{p}<0.05$ vs Cold, $n=6$.

Fig. 6. Effect of Atv on MAPK signal pathway in hearts of mice exposed to cold. Expression of p38 and p-p38 protein in hearts of Ctl, Cold and Atv groups. It indicated that cold exposure and Atv treatment had no effect on the expression of p38 and p-p38 protein in hearts; In addition, cold exposure as well as Atv had no effect on JNK and p-JNK protein expression in hearts as well. IOD of each protein band was determined with Image J software. Results are showed as mean \pm SEM. $n=6$.

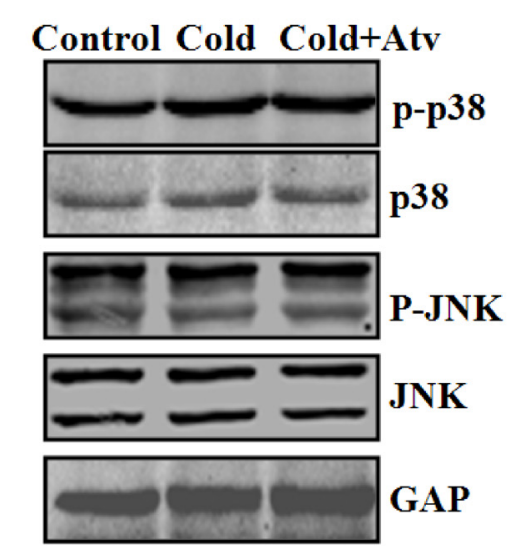

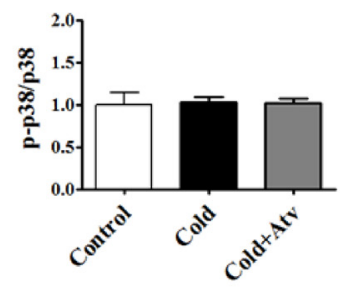

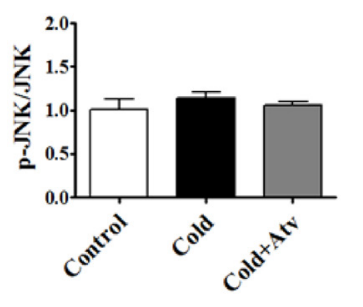

\section{Atv improved cardiac function of mice exposed to cold}

To explore effects of Atv on cardiac function of mice exposed to cold, ECG was performed after 8-week. Compared with Ctl, mice exposed to cold showed thickened ventricular wall and dilated ventricle that were reversed by Atv (Fig. 4A). Further analysis manifested that LVIDs/d increased after cold exposure which were reversed by Atv (Fig. 4B). EF decreased significantly after cold exposure while Atv was capable of improving EF. It indicated that Atv improved ejection function and alleviated cardiac remodeling.

\section{Atv decreased high blood pressure induced by cold exposure}

The blood pressure was recorded weekly from the $1^{\text {st }}$ week on. Figure 5 showed blood pressure fluctuation. There was no significant difference among groups until the $4^{\text {th }}$ week. On the $4^{\text {th }}$ week, systolic blood pressure (SBP) of mice exposed to cold was elevated significantly and Atv treatment repressed it. SBP of mice exposed to cold was increased other than mice of $\mathrm{Ctl}$ and Atv treatment groups from the $4^{\text {th }}$ week on. 


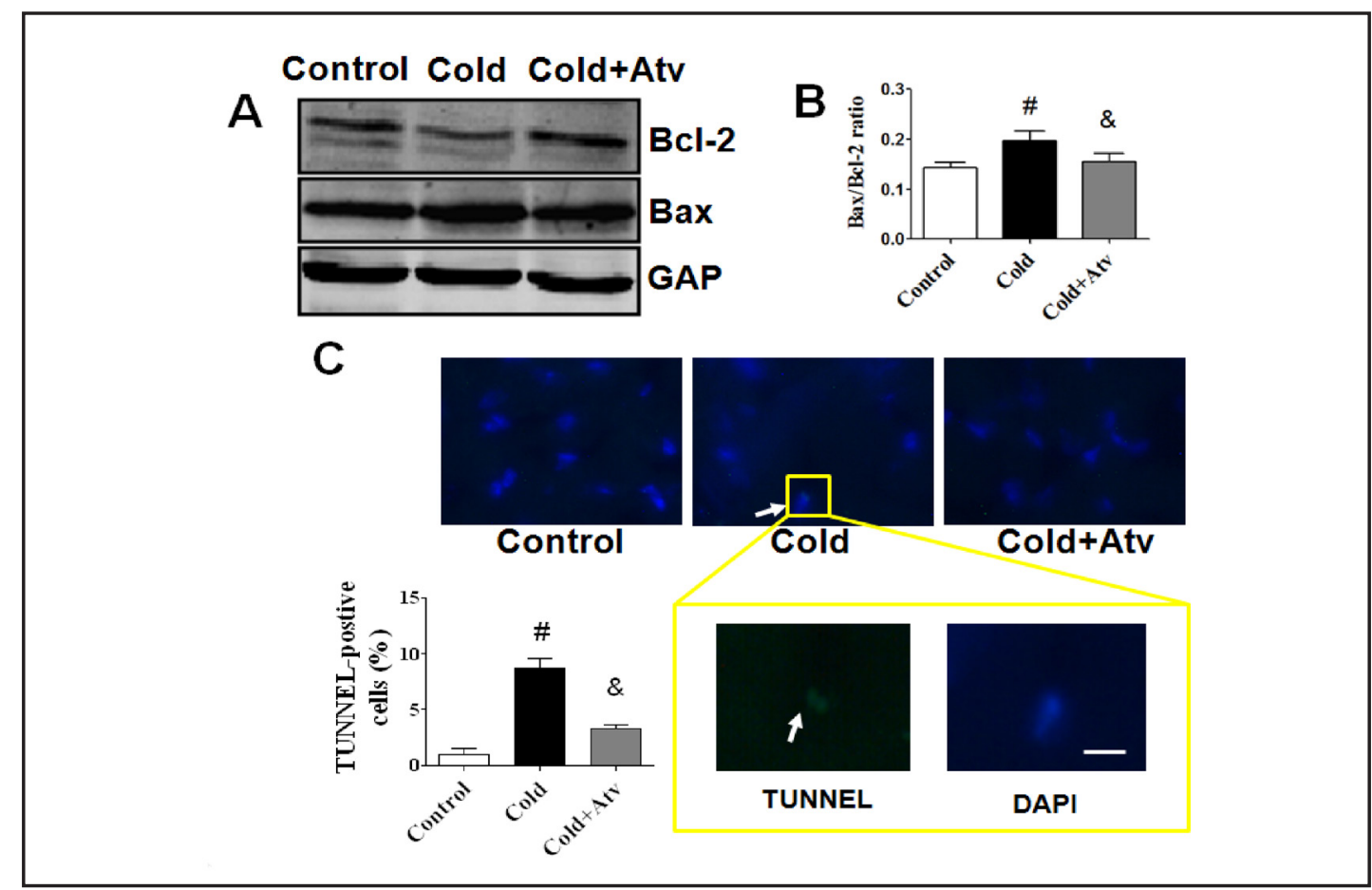

Fig. 7. Effect of Atv on apoptosis pathway in hearts of mice exposed to cold. A. Expression of apoptosis proteins in the groups of Ctl, Cold and Atv. It showed that cold exposure was capable of decreasing Bcl-2 in heart while Atv could increase the expression of Bcl-2 protein in hearts of mice exposed to cold significantly. However, Bax expression was increased after exposed to cold, which was reversed by Atv. B. Statistic analysis of Bcl-2/Bax level among three groups. C. TUNEL staining showed that Atv decreases cardiomyocytes apoptosis in the heart from cold-treated mice. IOD of each protein blot was determined with ImageJ software. Relative IOD was calculated as the ratio of protein IOD to GAPDH IOD. The scale bar: $10 \mu \mathrm{m}$. Results are showed as mean \pm SEM. \# $p<0.05$ vs Ctl, $\&$ p $<0.05$ vs Cold, $n=5$.

MAPK pathway was not involved in cardiac hypertrophy induced by cold exposure

To decipher potential mechanism of cardiac hypertrophy induced by cold exposure, Western blot was performed to determine proteins involved in several pathways. MAPK pathway was reported to be closely associated with cardiac hypertrophy under several conditions [23]. Therefore, components of MAPK pathway were determined (Fig. 6). These results showed that $\mathrm{p} 38$ and JNK expression did not change in cardiac hypertrophy induced by cold exposure. Likewise, Atv treatment did not affect p38 and JNK expression as well. It indicates that MAPK pathway is not involved in cold-induced myocardial hypertrophy.

Atv inhibited cardiomyocyte apoptosis induced by cold exposure through increasing Bcl2/Bax

Cardiomyocyte apoptosis was reported to be involved in cardiac hypertrophy [24]. Therefore, proteins associated with apoptosis pathway were determined. Our results showed that Bax expression was markedly increased in the heart from cold-treated mice, which can be abrogated by Atv treatment. However, Bcl-2 was downregulated after cold exposure while Atv increased Bcl-2 expression significantly (Fig. 7A). In agreement, TUNEL staining showed that Atv treatment suppressed the increase of apoptotic cardiomyocytes in cold-treated mice (Fig. 7B). It suggested that cold exposure could promote cardiomyocyte apoptosis by decreasing Bcl-2/Bax level while Atv inhibited it by counteracting this effect. 


\section{Cellular Physiology Cell Physiol Biochem 2017;41:286-295 \begin{tabular}{ll|l} 
and Biochemistry Published onIIne: January 30, 2017 & $\begin{array}{l}\text { (c) } 2017 \text { The Author(s). Published by S. Karger AG, Basel } \\
\text { www.karger.com/cpb }\end{array}$ \\
\hline
\end{tabular} \\ Liang et al.: Atorvastatin Inhibited Cold-Induced Hypertrophy}

\section{Discussion}

Our study determined structural and functional changes of heart after cold exposure as well as Atv administration. Our results showed that cold exposure could induce cardiac hypertrophy through increasing cardiomyocytes apoptosis while Atv alleviated hypertrophy through repressing apoptosis. Meanwhile, we observed that MAPK pathway was not involved in this condition.

It has been well documented that exposure to low temperature is an important cause for hypertrophic growth of hearts [6]. A series of studies showed that low ambient temperature might result in the elevation of blood pressure and the increase of cardiomyocytes size, and these pathological alterations will induce and even aggravate cardiac disorders [7-9]. How to prevent cold-induced myocardial hypertrophy is essential for the therapeutics of coldrelated diseases. Our previous study reported that Resveratrol inhibited cold-induced heart hypertrophy via suppressing miR-328 expression and apoptosis [7]. In this study, we detected the effects of Atv on blood pressure, cardiac function, cardiomyocyte hypertrophy, cardiac fibrosis and potential signaling pathways in cold-treated mice. Firstly, we observed that Atv treatment may attenuate the increase of heart weight/body weight index and heart weight/ tibial length index in cold-treated mice, which indicated Atv inhibited the hypertrophic growth of hearts. HE staining also displayed that the increased size of cardiomyocytes in mice exposed to cold temperature. This finding is consistent with previous studies [6-9]. Masson's trichrome staining further revealed that cold exposure resulted in cardiac fibrosis. Meanwhile, Transmission electron microscopy showed broken myofilament, swelling mitochondria and mitophagy in cardiomyocytes of mice exposed to cold, which were improved by Atv administration. But we did not investigate if there are functional changes of mitochondria in the heart of cold-treated mice, and examine the effects of Atv treatment on mitochondrial function in this study. This is a limitation of the present study. The data described above showed the evidence of structural damage of heart from cold-treated mice.

It is well known that cardiac structure damage usually led to the dysfunction of hearts. Therefore, we also detected cardiac functions among three groups, and the results confirmed that cold exposure contributed to the decline of cardiac functions. Atv treatment improves cardiac functions of cold-treated mice. Furthermore, western blot showed decreased antiapoptotic protein after cold exposure which was reversed by Atv. Here, in our study, we manifested the anti-apoptotic role of Atv in cardiac hypertrophy induced by cold exposure. This is consistent with study of Song et al. [25].

Considerable pathways such as oxidant stress are involved in pathophysiological regulation of cardiomyocytes besides MAPK and apoptosis pathways [26]. Thus, Atv might play its cardioprotective role in CVD treatment by mediating multiple pathways. A study concerning effect of Atv on heart failure induced by infarction manifested that Atv could inhibit cardiomyocytes apoptosis via mediating ER stress [25]. The direct evidence concluding role of Atv in hypertrophic heart showed that it could attenuate cardiac hypertrophy through regulating Akt/GSK-3 $\beta$ pathway [27]. Previous study suggested that Atv was capable of alleviating calcification of aortic valves induced by hypercholesterolemia and later investigation demonstrated that this effect was mediated by inhibiting Lrp $5 / \beta$-catenin pathway [28]. Cardiac hypertrophy induced by hypertension is frequently accompanied with autophagy. Wang et al. suggested that Atv regulated cardiac autophagy through mediating Akt/mTOR pathway, thus protecting cardiac structure and function from hypertension [29]. Consistently, Liu et al. concluded that Atv could regulate $\beta$-catenin-autophagy pathway [30]. In hypoxic pulmonary hypertension, Atv mitigated this condition by inhibiting RhoA/Rho kinase pathway in pulmonary arterial cells [31]. Afterwards, Yan Zhang drew the same conclusion and suggested a role of NF- $\mathrm{KB}$ in pulmonary hypertension [32]. Moreover, Atv could modulate translocation of $G$ protein subunits, thus regulating $G$ protein associated signaling [33]. These findings provided the novel research scope associated with Atv.

In conclusion, various factors are involved in CVD and Atv mitigates them via various pathways. Thus, it is clinically significant to give insight into these pathways and to find 
dominant ones. Notably, although Atv can protect heart from CVD, difference in dosage and administration approaches will result in different effect. Thus, specific medication is required for each kind of CVD.

\section{Conclusion}

Atorvastatin is capable of attenuating cardiac hypertrophy induced by cold exposure by repressing cardiomyocytes apoptosis. Our study provides a novel insight into the mechanism and therapeutic potential of Atv in cold-induced myocardial hypertrophy.

\section{Acknowledgements}

This study was supported by the Natural Science Foundation of China (81470463/81201875), University Nursing Program for Young Scholars with Creative Talents in Heilongjiang Province (2016LCZX27) and the Natural Science Foundation of Heilongjiang Province (LC2015027).

\section{Disclosure Statement}

The authors had no conflicts of interest to declare in relation to this article.

\section{References}

1 Frey N, Katus HA, Olson EN, Hill JA: Hypertrophy of the heart: A new therapeutic target? Circulation 2004;109:1580-1589.

- Rosca MG, Tandler B, Hoppel CL: Mitochondria in cardiac hypertrophy and heart failure. J Mol Cell Cardiol 2013;55:31-41.

-3 Yamori Y, Mori C, Nishio T, Ooshima A, Horie R, Ohtaka M, Soeda T, Saito M, Abe K, Nara Y, Nakao Y, Kihara M: Cardiac hypertrophy in early hypertension. Am J Cardiol 1979;44:964-969.

-4 Dekker LR, Rademaker H, Vermeulen JT, Opthof T, Coronel R, Spaan JA, Janse MJ: Cellular uncoupling during ischemia in hypertrophied and failing rabbit ventricular myocardium: Effects of preconditioning. Circulation 1998;97:1724-1730.

5 Rader F, Sachdev E, Arsanjani R, Siegel RJ: Left ventricular hypertrophy in valvular aortic stenosis: Mechanisms and clinical implications. Am J Med 2015;128:344-352.

6 Cai B, Gong D, Chen N, Li J, Wang G, Lu Y, Yang B: The negative inotropic effects of homocysteine were prevented by matrine via the regulating intracellular calcium level. Int J Cardiol 2011;150:113-115.

7 Yin K, Zhao L, Feng D, Ma W, Liu Y, Wang Y, Liang J, Yang F, Bi C, Chen H, Li X, Lu Y, Cai B: Resveratrol attenuated low ambient temperature-induced myocardial hypertrophy via inhibiting cardiomyocyte apoptosis. Cell Physiol Biochem 2015;35:2451-2462.

8 Lu S, Xu D: Cold stress accentuates pressure overload-induced cardiac hypertrophy and contractile dysfunction: Role of trpv1/ampk-mediated autophagy. Biochem Biophys Res Commun 2013;442:8-15.

9 Bello Roufai M, Li H, Sun Z: Heart-specific inhibition of protooncogene c-myc attenuates cold-induced cardiac hypertrophy. Gene Ther 2007;14:1406-1416.

$>10$ Sheth T, Nair C, Muller J, Yusuf S: Increased winter mortality from acute myocardial infarction and stroke: The effect of age. J Am Coll Cardiol 1999;33:1916-1919.

11 Fu S, Cao Y, Li Y: [epidemiological study of hypertension in heilongjiang province]. Zhonghua Nei Ke Za Zhi 2002;41:114-116.

-12 Fujiwara T, Kawamura M, Nakajima J, Adachi T, Hiramori K: Seasonal differences in diurnal blood pressure of hypertensive patients living in a stable environmental temperature. J Hypertens 1995;13:1747-1752.

-13 Wollert KC, Drexler H: Regulation of cardiac remodeling by nitric oxide: Focus on cardiac myocyte hypertrophy and apoptosis. Heart Fail Rev 2002;7:317-325. 


\section{Cellular Physiology Cell Physiol Biochem 2017;41:286-295

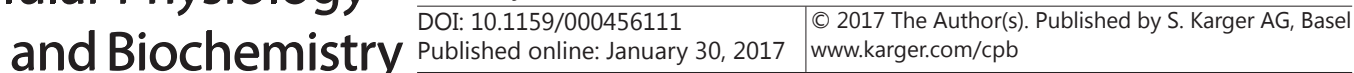

Liang et al.: Atorvastatin Inhibited Cold-Induced Hypertrophy

14 Iwai N, Shimoike H, Kinoshita M: Cardiac renin-angiotensin system in the hypertrophied heart. Circulation 1995;92:2690-2696.

15 Schlaich MP, Kaye DM, Lambert E, Sommerville M, Socratous F, Esler MD: Relation between cardiac sympathetic activity and hypertensive left ventricular hypertrophy. Circulation 2003;108:560-565.

16 Irukayama-Tomobe Y, Miyauchi T, Sakai S, Kasuya Y, Ogata T, Takanashi M, Iemitsu M, Sudo T, Goto $\mathrm{K}$, Yamaguchi I: Endothelin-1-induced cardiac hypertrophy is inhibited by activation of peroxisome proliferator-activated receptor- $\alpha$ partly via blockade of c-jun nh2-terminal kinase pathway. Circulation 2004;109:904-910.

17 Canas JA, Ross JL, Taboada MV, Sikes KM, Damaso LC, Hossain J, Caulfield MP, Gidding SS, Mauras N: A randomized, double blind, placebo-controlled pilot trial of the safety and efficacy of atorvastatin in children with elevated low-density lipoprotein cholesterol (ldl-c) and type 1 diabetes. Pediatr Diabetes 2015;16:79-89.

18 Podszun MC, Grebenstein N, Spruss A, Schlueter T, Kremoser C, Bergheim I, Frank J: Dietary alphatocopherol and atorvastatin reduce high-fat-induced lipid accumulation and down-regulate cd36 protein in the liver of guinea pigs. J Nutr Biochem 2014;25:573-579.

19 Yi R, Xiao-Ping G, Hui L: Atorvastatin prevents angiotensin ii-induced high permeability of human arterial endothelial cell monolayers via rock signaling pathway. Biochem Biophys Res Commun 2015;459:94-99.

20 Fang D, Yang S, Quan W, Jia H, Quan Z, Qu Z: Atorvastatin suppresses toll-like receptor 4 expression and nfkappab activation in rabbit atherosclerotic plaques. Eur Rev Med Pharmacol Sci 2014;18:242-246.

-21 Cai B, Wang G, Chen N, Liu Y, Yin K, Ning C, Li X, Yang F, Wang N, Wang Y, Pan Z, Lu Y: Bone marrow mesenchymal stem cells protected post-infarcted myocardium against arrhythmias via reversing potassium channels remodelling. J Cell Mol Med 2014;18:1407-1416.

22 Yamaguchi M, Tsuruda T, Watanabe Y, Onitsuka H, Furukawa K, Ideguchi T, Kawagoe J, Ishikawa T, Kato J, Takenaga M, Kitamura K: Reduced fractional shortening of right ventricular outflow tract is associated with adverse outcomes in patients with left ventricular dysfunction. Cardiovascular Ultrasound 2013;11:18.

23 Zhang W, Elimban V, Nijjar MS, Gupta SK, Dhalla NS: Role of mitogen-activated protein kinase in cardiac hypertrophy and heart failure. Exp Clin Cardiol 2003;8:173-183.

24 Teiger E, Than VD, Richard L, Wisnewsky C, Tea BS, Gaboury L, Tremblay J, Schwartz K, Hamet P: Apoptosis in pressure overload-induced heart hypertrophy in the rat. J Clin Invest 1996;97:2891-2897.

25 Song XJ, Yang CY, Liu B, Wei Q, Korkor MT, Liu JY, Yang P: Atorvastatin inhibits myocardial cell apoptosis in a rat model with post-myocardial infarction heart failure by downregulating er stress response. Int J Med Sci 2011;8:564-572.

26 Cai B, Pan Z, Liu Y, Chen N, Lu Y: Arrhythmogenic potential of oxidative stress in atrial myocytes. Int J Cardiol 2011;150:219-221.

27 Planavila A, Rodriguez-Calvo R, Palomer X, Coll T, Sanchez RM, Merlos M, Laguna JC, Vazquez-Carrera M: Atorvastatin inhibits gsk-3beta phosphorylation by cardiac hypertrophic stimuli. Biochim Biophys Acta 2008;1781:26-35.

-28 Rajamannan NM, Subramaniam M, Caira F, Stock SR, Spelsberg TC: Atorvastatin inhibits hypercholesterolemia-induced calcification in the aortic valves via the $\operatorname{lrp} 5$ receptor pathway. Circulation 2005;112:1229-234.

29 Wang W, Wang H, Geng QX, Wang HT, Miao W, Cheng B, Zhao D, Song GM, Leanne G, Zhao Z: Augmentation of autophagy by atorvastatin via akt/mtor pathway in spontaneously hypertensive rats. Hypertens Res 2015;38:813-820.

-30 Liu D, Cui W, Liu B, Hu H, Liu J, Xie R, Yang X, Gu G, Zhang J, Zheng H: Atorvastatin protects vascular smooth muscle cells from tgf-beta1-stimulated calcification by inducing autophagy via suppression of the betacatenin pathway. Cell Physiol Biochem 2014;33:129-141.

-31 Dai L, Wu S: [atorvastatin attenuates hypoxic pulmonary hypertension in rats by inhibiting rhoa/rho kinase pathway]. Zhong Nan Da Xue Xue Bao Yi Xue Ban 2011;36:58-63.

-32 Zhang Y, Dai L, Wu S, Chen P, Zhao S: Atorvastatin attenuates involvement of rhoa/rho-kinase pathway and nf-kappab activation in hypoxic pulmonary hypertensive rats. Chin Med J (Engl) 2014;127:869-872.

33 Schmechel A, Grimm M, El-Armouche A, Hoppner G, Schwoerer AP, Ehmke H, Eschenhagen T: Treatment with atorvastatin partially protects the rat heart from harmful catecholamine effects. Cardiovasc Res 2009;82:100-106. 